\title{
High power microwave diagnostic for the fusion energy experiment ITER
}

\author{
Korsholm, Søren Bang; Leipold, Frank; Goncalves, B.; Gutierrez Espinoza, Heidi Estibaliz; Jensen, \\ Thomas; Jessen, Martin; Klinkby, Esben Bryndt; Larsen, Axel Wright; Naulin, Volker; Nielsen, Stefan \\ Kragh \\ Total number of authors: \\ 17
}

Published in:

2016 41st International Conference on Infrared, Millimeter, and Terahertz waves (IRMMW-THz)

Link to article, DOI:

10.1109/IRMMW-THz.2016.7758537

Publication date:

2016

Document Version

Peer reviewed version

Link back to DTU Orbit

Citation (APA):

Korsholm, S. B., Leipold, F., Goncalves, B., Gutierrez Espinoza, H. E., Jensen, T., Jessen, M., Klinkby, E. B., Larsen, A. W., Naulin, V., Nielsen, S. K., Nonbøl, E., Oosterbeek, H., Rasmussen, J., Salewski, M., Sanchez, P., Pedersen, M. S., \& Taormina, A. (2016). High power microwave diagnostic for the fusion energy experiment ITER. In 2016 41st International Conference on Infrared, Millimeter, and Terahertz waves (IRMMW-THz) IEEE. International Conference on Infrared, Millimeter and Terahertz Waves https://doi.org/10.1109/IRMMW$\mathrm{THz} .2016 .7758537$ 


\title{
High Power Microwave Diagnostic for the Fusion Energy Experiment ITER
}

\author{
S.B. Korsholm ${ }^{1}$, F. Leipold ${ }^{1}$, B. Gonçalves ${ }^{2}$, H.E. Gutierrez ${ }^{1}$, T. Jensen ${ }^{1}$, M. Jessen ${ }^{1}$, E.B. Klinkby ${ }^{5}$,
} A.W. Larsen ${ }^{1}$, V. Naulin ${ }^{1}$, S.K. Nielsen ${ }^{1}$, E. Nonbøl' ${ }^{5}$, H. Oosterbeek ${ }^{4}$, J. Rasmussen ${ }^{1}$, M. Salewski ${ }^{1}$, L. Sanchez $^{3}$, P. Sanchez ${ }^{3}$, M. Stejner ${ }^{1}$, and A. Taormina ${ }^{1}$

${ }^{1}$ Technical University of Denmark, Department of Physics, 2800 Kgs. Lyngby, Denmark

${ }^{2}$ Instituto de Plasmas e Fusão Nuclear, Instituto Superior Técnico, Univ. Lisboa, Portugal

${ }^{3}$ Fusion for Energy, 08019 Barcelona, Spain

${ }^{4}$ Eindhoven University of Technology, 5600 MB Eindhoven, The Netherlands

${ }^{5}$ Technical University of Denmark, Center for Nuclear Technologies, 4000 Roskilde, Denmark

\begin{abstract}
Microwave diagnostics will play an increasingly important role in burning plasma fusion energy experiments like ITER and beyond. The Collective Thomson Scattering (CTS) diagnostic to be installed at ITER is an example of such a diagnostic with great potential in present and future experiments. The ITER CTS diagnostic will inject a $1 \mathrm{MW} 60 \mathrm{GHz}$ gyrotron beam into the ITER plasma and observe the scattering off fluctuations in the plasma - to monitor the dynamics of the fast ions generated in the fusion reactions.
\end{abstract}

\section{INTRODUCTION}

$\mathrm{T}$ HE international fusion energy experiment ITER encompasses a range of diagnostics for optimizing the operation and measuring the performance of the machine. The full diagnostic suite is based on diagnostic systems that have been tested and used on smaller existing experiments. They are now being implemented in new incarnations adapted to an environment with intense neutron and gamma radiation not previously experienced in thermonuclear fusion experiments. The neutrons are born at energies of $14 \mathrm{MeV}$, and the power load on the vacuum vessel walls may exceed 1 $\mathrm{MW} / \mathrm{m}^{2}$. The diagnostic system designs are also being prepared for the future burning plasma fusion reactors. Microwave diagnostics in general are among the few types of diagnostics that will be appropriate for fusion power plants [1, 2], and thus the development of these for ITER carry a particular importance. The Collective Thomson Scattering (CTS) diagnostic [3] is such a microwave based system that enables measurements of the ion velocity distribution function - both temporally $(\sim 100 \mathrm{~ms})$ and spatially $(\sim 20-50 \mathrm{~cm})$ resolved - in ITER.

The principle of the CTS diagnostic is that a quasi-optical mirror captures part of the radiation that is scattered due to the interaction between an injected powerful source beam (typically a gyrotron in the $100 \mathrm{GHz}$ and $100 \mathrm{~s} \mathrm{~kW}$ range) and microscopic fluctuations in the plasma. These microscopic fluctuations are mainly caused by the dynamics of the ions in the plasma, and thus one may deduce a $1 \mathrm{D}$ projection of the ion velocity distribution function. From this, one may potentially obtain measurements of the bulk ion temperature, bulk ion rotation, bulk ion fuel composition, and the fast ion velocity distribution $[4,5,6]$. In ITER the primary role of the CTS diagnostic is measurements of the $1 \mathrm{D}$ velocity distribution of the fusion born alpha particles. This is an essential physics measurement on ITER - being the first real alpha producing fusion experiment.

\section{RESUlts}

The design of the ITER CTS diagnostic is an elaborate process due to the many restrictions, boundary conditions, and requirements given by the nature of ITER as a nuclear facility. The design steps are prescribed and requirements for documentation of each design step are elaborate. This is a consequence of the challenging integration of the many ITER systems that mutually affect each other; not only between different diagnostic systems but not the least between diagnostics and other ITER systems or components. The boundary conditions do also affect some essential design decisions of the CTS diagnostic.

The integration of diagnostic and heating systems in ITER has been standardized by the introduction of drawers in portplugs. This facilitates the enormously elaborate integration process for the many systems. The CTS diagnostic has been allocated to a full drawer (approximately $0.5 \mathrm{~m}$ wide, $2 \mathrm{~m}$ tall

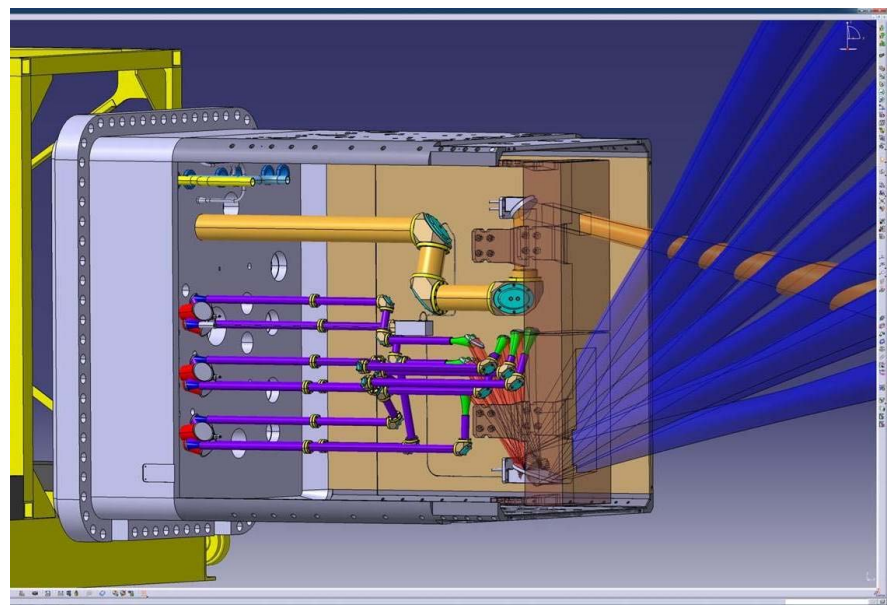

Fig. 1. CAD (CATIA) drawing showing the design of the beam paths in the ITER vacuum vessel port-plug \#12. The orange waveguide passes the $1 \mathrm{MW}$ gyrotron beam from the back of the port-plug to the front mirror facing the plasma (see text). The purple waveguides pass the receiver signals from the plasma facing mirrors to the end of the port-plug (see text).

and $2.5 \mathrm{~m}$ long) in the port-plug in equatorial port \#12 of ITER (the drawer and the port-plug is presented in Fig. 1). The sole occupancy of the drawer gives the advantage of more spatial freedom in the design process. However, being a microwave diagnostic operating at $60 \mathrm{GHz}$ and $1 \mathrm{MW}$ of injected gyrotron power, even this allocated space sets many design restrictions, due to the required size of e.g. the mirrors. 
A general challenge for any tokamak based system is the connection between the vacuum in-vessel and atmospheric pressure ex-vessel parts of the system. This accounts for e.g. electrical, optical, and quasi-optical connections. The portplug is closed and sealed by a port-plug closure plate, and all connections have to be located onto this with space allocations for installation and maintenance (See Fig. 2). This has posed particular challenges on the number of quasi-optical lines of the CTS diagnostic that can be fed through. The optimal solution that was identified and developed included three large diameter vacuum windows (largest standard components $160 \mathrm{~mm}$ diameter clear aperture). Through each of these, three

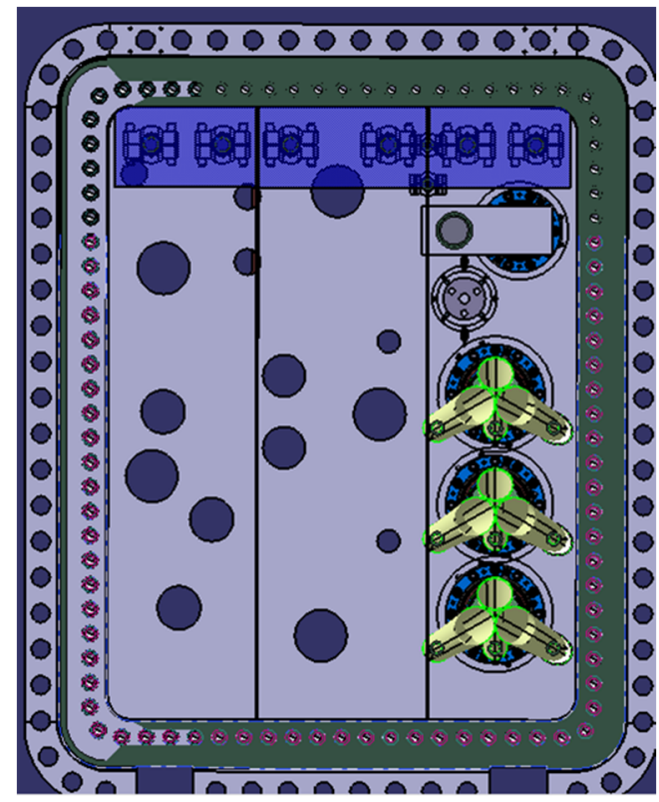

Fig. 2. CAD (CATIA) drawing showing the port-plug closure plate from outside the tokamak. The drawer to the right belongs to the CTS diagnostic system.

quasi-optical paths are focused by a set of two in-vessel and two ex-vessel mirrors per path coupling from the in-vessel waveguides to the ex-vessel waveguides. This limits the number of quasi-optical path lines to nine excluding the path of the probing gyrotron beam that has a completely separate path. The nine paths have to be shared between the receiver signals and all auxiliary lines. The latter includes possible calibration and microwave monitoring systems.

In Fig. 1 we show the high power gyrotron launcher waveguide in orange. The path of the gyrotron beam is separated from the paths of the receiver lines in order not to have any stray light directly in the port plug. The gyrotron beam is transmitted via four in-vessel mitre bends before it aims at the launcher mirror that directs and focuses the launcher beam into the tokamak vessel. The powerful gyrotron beam is not absorbed in the plasma (due to the deliberate choice of frequency) and the interaction between the beam and the plasma induces scattered radiation that is picked up by a number of receiver beams. The receiver beams (shown in blue in Fig. 1) are defined by a common plasma facing mirror and a series of individual mirrors (one for each beam) focusing the respective beam into the receiver waveguides shown in purple. The two-mirror in-vessel coupling units are shown at the back near the closure plate.

The ex-vessel transmission of the receiver signals is about $80 \mathrm{~m}$ to the neighboring diagnostic building, where cubicles are reserved for microwave radiometers to treat the received signals. Generally, the expected power level of the interesting parts of the scattered spectrum is in the order of $n \mathrm{~W}$, while the power level of the injected gyrotron power is in the order of 1 MW. This poses a challenge for the analysis of the CTS signals, but this is mastered in present day experiments.

The transmission of $1 \mathrm{MW}$ of gyrotron power from the gyrotron and into ITER is a particular challenge. As for the electron cyclotron heating gyrotrons for ITER, there will be a transmission loss in the $100+\mathrm{m}$ transmission line from the gyrotron to the ITER vessel. This will amount to about 100$150 \mathrm{~kW}$. However, the particular challenge for the ITER CTS gyrotron is that the standard ITER magnetic field matches the fundamental resonance for the gyrotron frequency in the middle of the port-plug. Hence, some attention should be devoted to the issue of risk of arcing, breakdown and plasma generation in the transmission line in the port-plug. Dedicated mock-up experiments are planned and possible mitigation actions are being investigated.

Presently the diagnostic design has passed the System Level Design and is progressing well towards a design that will be evaluated in terms of port integration (see Fig. 1), neutronics calculations, and diagnostic performance. The provisional expectations for the diagnostic performance will be presented for a system with the before-mentioned $1 \mathrm{MW} 60 \mathrm{GHz}$ gyrotron and seven lines of receiver views.

\section{SUMMARY}

The contribution describes the current state of the design of the CTS diagnostic system to be installed at the ITER fusion energy experiment. The CTS diagnostic will be key to the measurement of dynamics of the fusion born alpha particles.

\section{ACKNOWLEDGEMENT}

The work leading to this publication has been funded partially by Fusion for Energy under F4E-FPA-393. This publication reflects the views only of the author(s), and Fusion for Energy cannot be held responsible for any use which may be made of the information contained therein. IPFN activities received financial support from "Fundação para a Ciência e Tecnologia” through project UID/FIS/50010/2013.

\section{REFERENCES}

[1]. M. Salewski et al, "Investigation of first mirror heating for the collective Thomson scattering diagnostic in ITER" Rev Sci Instrum, vol. 79, 10E729, 2008.

[2]. F. Leipold et al, "Antenna design for fast ion collective Thomson scattering diagnostic for the international thermonuclear experimental reactor, Rev Sci Instrum, vol. 80, 093501, 2009.

[3]. S.B. Korsholm et al, "Collective Thomson scattering capabilities to diagnose fusion plasmas," Nucl. Instr. Meth. Phys. Res. A, vol. 623, pp. 677680, 2010.

[4]. S.B. Korsholm et al, "Measurements of Intrinsic Ion Bernstein Waves in a Tokamak by Collective Thomson Scattering," Phys. Rev. Lett., vol. 106, 165004, 2011.

[5]. M. Stejner et al, "The prospect for fuel ion ratio measurements in ITER by collective Thomson scattering," Nucl. Fusion, vol. 52, 023011, 2012.

[6]. M. Salewski et al, "Comparison of collective Thomson scattering signals due to fast ions in ITER scenarios with fusion and auxiliary heating," Plasma Phys. Control. Fusion, vol. 51, 035006, 2009. 